\title{
Genetic Improvement of Grain Quality Promoted by High and New Technology in Rice
}

\author{
Bo Peng ${ }^{1}$, Jun $\mathrm{Li}^{1}$, Dong-Yan Kong ${ }^{1}$, Lu-Lu He ${ }^{1}$, Meng-Ge $\mathrm{Li}^{1}$, Tondi-Yacouba Nassirou ${ }^{2}, \mathrm{Yu}_{\text {Peng }}{ }^{3}$, \\ Xiao-Hua Song ${ }^{4}$, Juan Peng ${ }^{5}$, Yue Jiang ${ }^{1}$, Yan-Fang Sun ${ }^{1}$, Rui-Hua Pang ${ }^{1}$, Qing-Qing Xin ${ }^{1}$, Yu-Chen Liu ${ }^{6}$, \\ Gui-Ying Guo ${ }^{4}$, Jin-Tiao Li ${ }^{1}$, Quan-Xiu Wang ${ }^{1}$, Shi-Zhi Song ${ }^{4}$, Bin Duan ${ }^{4} \&$ Hong-Yu Yuan ${ }^{1}$ \\ ${ }^{1}$ College of Life Sciences and Institute for Conservation and Utilization of Agro-bioresources in Dabie Mountains, \\ Xinyang Normal University, Xinyang, China \\ ${ }^{2}$ Plate Forme Agroécologique Multi Acteurs Raya Karkara Du Niger, Niamey, Niger \\ ${ }^{3}$ School of Science and Technology, Xinyang University, Xinyang, China \\ ${ }^{4}$ Xinyang Academy of Agricultural Science, Xinyang, China \\ ${ }^{5}$ Xinyang Station of Plant Protection and Inspection, Xinyang, China \\ ${ }^{6}$ College of International Education, Xinyang Normal University, Xinyang, China \\ Correspondence: Bo Peng, College of Life Sciences and Institute for Conservation and Utilization of \\ Agro-bioresources in Dabie Mountains, Xinyang Normal University, Xinyang 464000, China. E-mail: \\ pengbo@xynu.edu.cn \\ Tondi-Yacouba Nassirou, Plate Forme Agroécologique Multi Acteurs Raya Karkara Du Niger, BP: 11271, \\ Niamey, Niger. E-mail: tnassirou2009@gmail.com \\ Hong-Yu Yuan, College of Life Sciences and Institute for Conservation and Utilization of Agro-bioresources in \\ Dabie Mountains, Xinyang Normal University, Xinyang 464000, China. E-mail: yhongyu92@163.com
}

Received: September 15, 2018

Accepted: October 20, 2018

Online Published: December 15, 2018

doi:10.5539/jas.v11n1p81

URL: https://doi.org/10.5539/jas.v11n1p81

\begin{abstract}
Rice (Oryza sativa L.) is considered as one of the most important food crops all over the world. Because of the improvement of the actual living standards, there is more and more demand for improved grain quality. Although, the grain quality is a very complex quantitative trait in rice, it has great scientific significance and practical application value for the improvement of the quality of rice grain using high and up to date techniques. Here, we focus on the new advances in the application of the genetic improvement of grain quality by high throughput sequencing, genomic editing, near infrared spectroscopy, scanning electron microscopy, and other new techniques. Simultaneously, the application prospects of these high and up to date techniques have also been expected in the genetic improvement of rice quality. Thus, this will provide important information for genetic improvement of grain quality in rice breeding.
\end{abstract}

Keywords: Oryza sativa, high-throughput sequencing, genomic editing, near infrared spectroscopy, scanning electron microscopy, grain quality

\section{Introduction}

Rice (Oryza sativa L.) is, a staple food for more than 3 billion people in more than 100 countries and regions and considered as one of the most important food crops worldwide, and it provides about $40 \%$ of the total calorie intake for Chinese people (Kusano et al., 2015; Peng et al., 2016a). The continuous growth of the world's population and the improvement of people's living standards, make the genetic improvement of grain quality and popularization of the high-quality rice to become one of the key contents (Peng et al., 2014a). Therefore, increasing rice yield and improving grain quality are two important goals of plant basic science and applied scientific research (Zhou et al., 2017), and they are also the focus of concern for plant geneticists and plant breeders in China.

Grain quality is a very complex genetic trait in rice. Each quality trait is controlled by multiple genes, involving a variety of gene expression regulation and regulatory networks, and easily affected by external environmental factors, such as light, temperature and soil conditions (Chen et al., 2012; Zhang et al., 2016). At present, rice 
grain quality is generally considered the grain appearance and milling, eating, cooking, and nutritional qualities (Tsukaguchi et al., 2016; Pandey et al., 2012; Bhullar et al., 2013; Peng et al., 2014b). However, people in different countries and regions have different concerns about various grain quality traits in rice. For example, the developed countries require the good cooking and eating quality, while the people of India prefer the non-sticky rice and the people from Far East prefer the sticky and soft rice (Chen et al., 2012). For many people in the developing countries and regions, the rice is often the most important energy source (Peng et al., 2016b). Thus, it is of great scientific significance and application prospects to improve grain quality of rice.

Over the past decade, a series of new progress has been made in genetic improvement and related new technology research for rice grain quality. A large number of genes or quantitative trait loci related to grain quality traits were isolated and cloned in rice (Zhang et al., 2016; Peng et al., 2014b), important advances have also been made in the study of the functions of some important quality-related genes and their regulation. At the same time, some high and new technologies (such as the high-throughput sequencing technology and genome editing technology) have been widely used in the fields of agriculture, food industry, pharmaceuticals and medicine, especially in the process of improving and breeding the grain quality of major food crops. Detection and related analytical techniques are playing an increasingly important role in genetic improvement of rice grain quality. Here, we focus on the recent new advances in genome editing technology, high-throughput sequencing technology, near-infrared spectroscopy, scanning electron microscopy, and other high and new technologies in the application of grain quality improvement in rice. The application prospects of modern detection and analysis techniques in rice quality improvement also discussed, this will provide an important reference for the genetic improvement of the grain quality of major grain crops and the cultivation of new varieties.

\section{Application of High-Throughput Sequencing Technology in Genetic Improvement of Rice Grain Quality}

High-throughput sequencing technology, also known as next generation sequencing technology, is characterized by low sequencing cost and large amount of data output. It is a revolutionary technological innovation in the process of nucleic acid research (Ansorge, 2009; Liu et al., 2016). A large number of single nucleotide polymorphisms, insertion/deletion and structural variability sites in rice genome can be obtained by high throughput sequencing technology using the natural population, genetic mapping population or mutant individuals in rice. On the basis of these genetic variations, a large number of molecular markers can be obtained by linkage analysis, association analysis, comparative study and related bioinformatics analysis (Subbaiyan et al., 2012; Araikichise et al., 2011). At present, high-density genetic maps of rice has been constructed by high-throughput sequencing technology (Wang et al., 2011; Yu et al., 2011; Gao et al., 2016), and it contains a large number of molecular markers, which have been applied to the location quantitative trait loci (QTL) of rice quality and yield or the mining of candidate genes (Xu et al., 2015). This will promote the genetic improvement of existing cultivated rice, and then accelerate the cultivation of new varieties with high quality in rice (Figure 1).

At the moment, high-throughput sequencing technology plays an important role in the location and exploration of rice grain quality related QTLs or genes (Huang et al., 2012a; Xu et al., 2015). It has been showed by various studies that it is possible to construct genetic maps (Huang et al., 2012a; Gao et al., 2015), establish its database of single nucleotide polymorphism (SNP) and Insertions or Deletions (InDel) mutations (Li et al., 2014a; Tatarinova et al., 2016), through high-throughput genome re-sequencing in rice. These findings provide a large number of polymorphic molecular markers for the genetic improvement of grain quality and promote the molecular breeding for rice quality. For example, through a large-scale sequencing of 517 local rice varieties and 950 cultivated rice varieties, the genome wide association analysis (GWAS) was applied to study the grain quality and yield of these rice varieties. Similarly, a high density haplotype map of rice has been constructed to develop more than one million SNPs markers, and lots of new QTLs related to rice grain quality was also discovered (Huang et al., 2010, 2012b; Xu et al., 2015). Moreover, using 150 recombinant inbred populations, a genetic map containing 2334 SNPs markers was constructed by high throughput sequencing technology. Dozens of QTLs related to the important agronomic traits were identified in rice, and multiple QTLs controlling grain length and grain width were located in smaller intervals witch make it easier to analyze directly the candidate genes (Wang et al., 2011). Likewise, high throughput sequencing technology was used to detect a number of QTLs that was closely related to rice grain quality in 241 recombinant inbred lines from zhensan 97 and Minghui 63, and verified the 3 main QTLs (GS3, GW5 and $O s C 1$ ) controlling rice grain quality traits (Yu et al., 2011). Therefore, high throughput sequencing technology, especially the application of re-sequencing technology to construct the rice genetic map, GWAS analysis, and even the genetic research of mutant plants in rice, will promote the discovery of QTLs or functional genes related to the important traits of rice grain quality, and then accelerate the research and extensive application of molecular breeding for rice grain quality. 
Compared with traditional technology, high throughput sequencing technology has many advantages: short time, high efficiency and low cost; the number of SNPs, InDels or structural variation (SVs) is large, and the location of QTLs or functional genes is more accurate with a smaller interval of location; the isolation and cloning of functional genes and molecular marker assisted selection in the later period bring great convenience to molecular breeding in rice (Shapter et al., 2013; Huang et al., 2015). Therefore, high throughput sequencing technology has been widely used in rice genome sequencing, small RNAs sequencing, transcriptional sequencing, epigenetic sequencing and other fields, which has brought great opportunities for the genetic improvement of rice grain quality. Some new technologies, such as degradation group sequencing, chromatin immunoprecipitation DNA sequencing and DNA methylation sequencing technology accompanied by high throughput sequencing technology will provide new ideas for genetic improvement and molecular breeding of rice grain quality. However, it should be clear to realize the massive sequence data accompanied by high throughput sequencing technology and how to analyze these sequence information is still a great challenge (Zhou et al., 2017). How to fully explore the biological laws and its significance behind a large number of sequencing data as well as the classification, sorting and access of the data remains to be further solved.

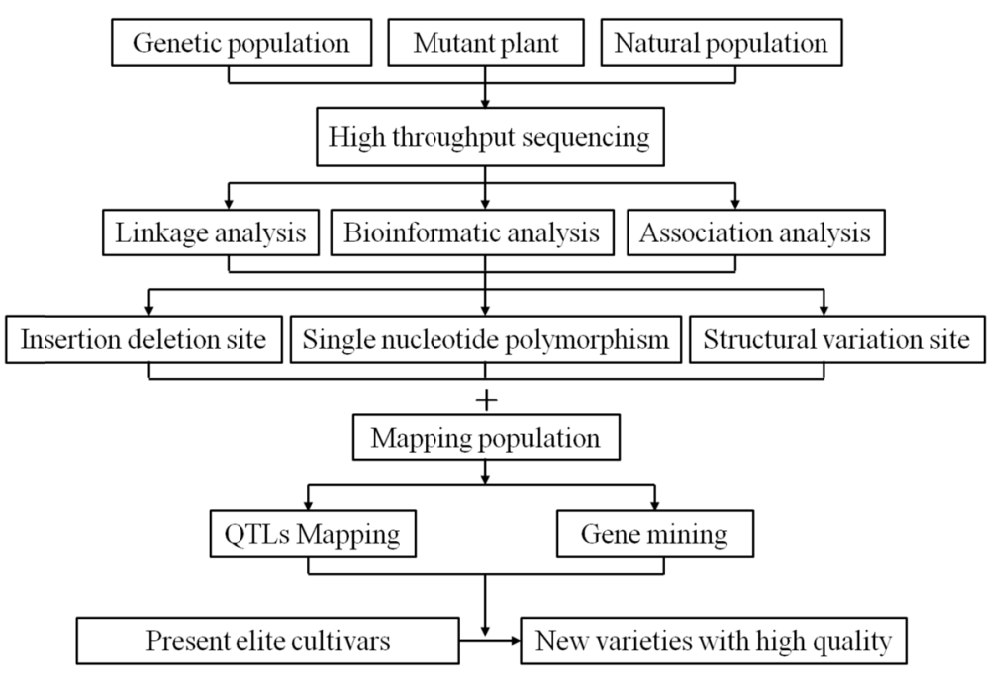

Figure 1. High throughput sequencing for gene discovering and grain quality improvement in rice

\section{Application of Genome Editing Technology in Genetic Improvement of Rice Grain Quality}

Genomic editing based on sequence-specific nucleases (SSNs) has become one of the most effective new technologies for crop genetic improvement in recent years (Samanta et al., 2016; Liu et al., 2017). At present, many different types of SSNs have been developed for genome editing, including zinc finger nuclease (ZFNs), transcriptional activator-like effector nuclease (TALENs) and the clustered regularly interspaced short palindromic repeat (CRISPR/Cas9) and so on. ZFNs, TALENs and CRISPR/Cas9 technologies are based on their own endonuclease activity, causing DNA double strand breakage at the target DNA sequence, and introducing mutations through homologous directed repair or non-homologous end joining (NHEJ) (Peng et al., 2016a). Then, we could successfully implement gene editing, such as gene knockout, gene insertion, point mutation, gene modification or transcriptional activation in different organisms (Figure 2).

Genome editing technology has developed rapidly in the past decade, and this technology based on SSNs has been widely used in the field of basic research and application of plant (Peng et al., 2017). For example, the use of ZFNs, TALENs, and CRISPR/Cas9 technologies have successfully achieved gene knockouts, point mutations, or modification of specific genes for a variety of plants (Liu et al., 2017), including important crops such as Oryza sativa, Zea mays, Triticum aestivum, Hordeum vulgare and soybean (Li et al., 2017; Wendt et al., 2013; Liang et al., 2014; Wang et al., 2014; Shan et al., 2015; Li et al., 2012). Among them, TALEN technology has been applied to the genetic breeding of fragrant rice (Shan et al., 2013, 2015; Birla et al., 2017). This technology provides a new strategy for rice breeders to improve grain quality of fragrant rice. The previous study found that the Badh2 gene was located on the eighth chromosome of rice genome was closely related to the aroma of rice. The Badh2 protein encoded by the Badh2 gene in the non fragrant rice has the activity of betaine dehydrogenase. 
The loss of betaine dehydrogenase activity in mutant types leads to the accumulation of 2-acetyl-1-pyrroline (2-AP) in fragrant rice (Kovach et al., 2009; He et al., 2015). Therefore, TALEN technology was used to introduce fixed point mutation at the location of Badh2 gene in non fragrant rice varieties (Shan et al., 2015), resulting in a significant increase in 2-AP content in the non fragrant of $\mathrm{T}_{1}$ generation, and could be stable to the $\mathrm{T}_{2}$ generation in rice individuals, and then the non fragrant rice could produce the same aroma.

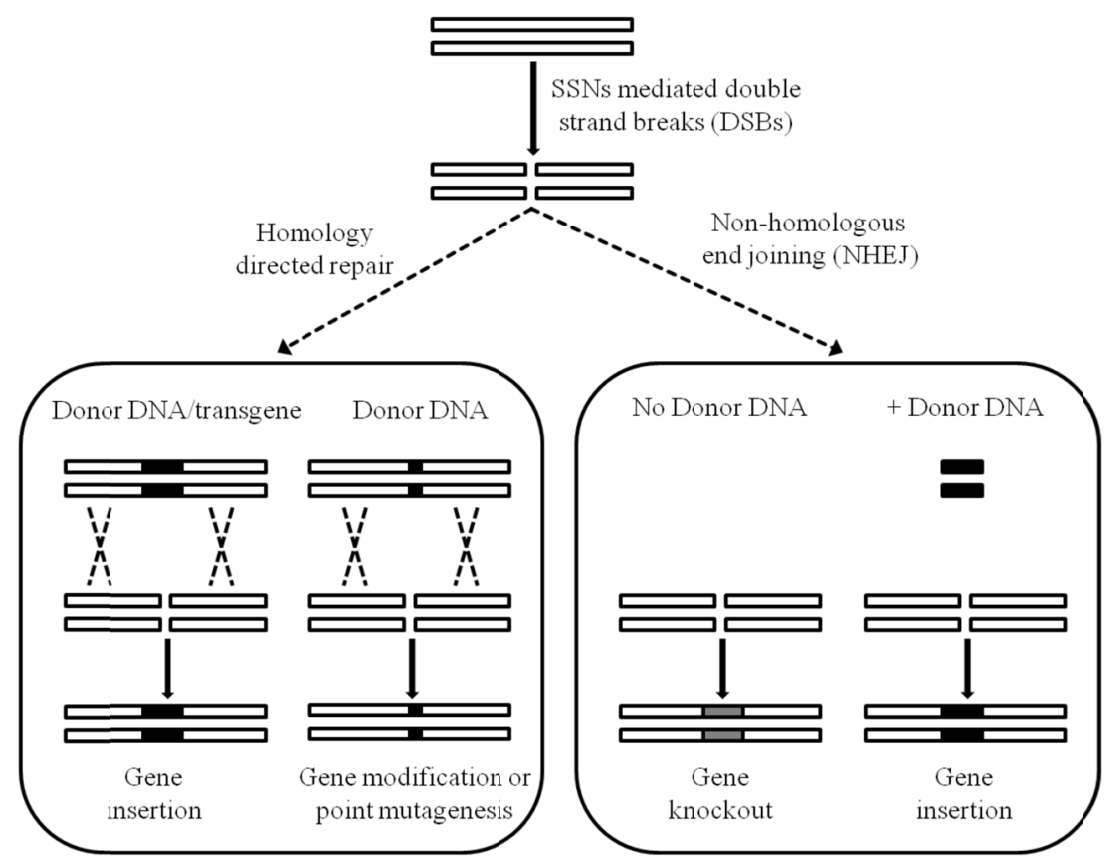

Figure 2. Overview of possible outcomes from genome editing using sequence-specific nucleases

Compared with ZFNs and TALENs technology, CRISPR/Cas9 technology was simple operation, low cost, high efficiency, and could obtain stable genetic progeny genome editing plants, and also could be used to modify or edit genes at different locations in the rice genome simultaneously (Xu et al., 2016, 2017; Li et al., 2017; Zhou et al., 2014; Johnson et al., 2015). Therefore, CRISPR/Cas9 technology has been widely applied in the research of rice grain quality and the quality related traits (Ma et al., 2015; Li et al., 2016, 2017; Su et al., 2012; Feng et al., 2013; Zhang et al., 2014; Miao et al., 2013; Xu et al., 2015; Shen et al., 2016; Zhou et al., 2014; Wang et al., 2015; Mao et al., 2013). For example, the main effect gene Waxy (GBSS) controlling the amylose content in rice grain was mutated by CRISPR/Cas9 technology and the amylose content was reduced from $14.6 \%$ to $2.6 \%$ in the mutated plants, which was similar to the amylose content in the glutinous rice (Ma et al., 2015). The $S B E I$ and SBEIIb genes in rice were mutated by CRISPR/Cas9 technology, the amylose content and the resistant starch content of the corresponding mutants increased by $25 \%$ and $9.8 \%$ respectively, and then the fine structure and nutritive properties of the starch were also changed. Similarly, CRISPR/Cas9 technology has also been reported for the successful directed editing of genes related to the appearance quality of rice grain and its yield traits (such as Gn1a, DEP1, GS3 and IPA1) (Li et al., 2016). YSA (Young seedling albino), CAO1 and LAZY1 related to chlorophyll synthesis in rice was directionally mutated by CRISPR/Cas9 technology, which led to the early screening and identification of false hybrids in hybrid rice seeds could be quickly and effectively (Su et al., 2012; Feng et al., 2013; Zhang et al., 2014; Miao et al., 2013). Hd2, Hd4 and $H d 5$ genes were mutated simultaneously by CRISPR/Cas 9 technology in 14 of the 18 corresponding transgenic families, and the efficiency of multi gene simultaneous editing was as high as $77.8 \%$. Then the growth period of rice was significantly shortened, and the early maturing rice individuals were also obtained ( $\mathrm{Li}$ et al., 2017). Therefore, the genome editing technology, represented by CRISPR/Cas9 technology, plays an important role in the research process of rice grain quality and related traits, and it will greatly accelerate the genetic improvement of grain quality in rice.

Molecular design breeding for rice quality using genomic editing technology based on SSNs (including TALENs, ZFNs and CRISPR/Cas9) has obvious advantages over traditional hybrid breeding and modern transgenic (RNAi or genetic engineering) breeding in rice: (1) Edit target genes accurately; (2) There is no need for hybridization and backcross, and it is convenient and fast; (3) No need for large capital investment; (4) Individuals without 
selection markers can be obtained (Shan et al., 2015; Samanta et al., 2016; Li et al., 2017). Therefore, genome editing technology is a powerful tool in the field of crop genetic improvement and breeding. More and more genome editing technologies, such as TALENs, ZFNs and CRISPR/Cas9, will be applied to the genetic improvement of rice quality in the future so as to speed up the breeding process of new rice varieties with high quality.

\section{Application of Scanning Electron Microscope Technology in Genetic Improvement of Rice Grain Quality}

Compared with ordinary optical microscopes, scanning electron microscopy (SEM) has higher resolution rate and can analyze images of the microstructure of the surface and section of grain samples in rice. SEM is an intuitive and effective tool for detecting and analyzing the rice quality in recent years. Different types of japonica rice and fragrant rice and their corresponding rice quality have their specific starch grain shape and arrangement, and their quality could be identified by SEM (Peng et al., 2016b; Kang et al., 2007). Currently, SEM has been widely used in studies of physiological characteristics, morphological structure, and grain quality improvement in rice (Li et al., 2014b; Zhou et al., 2010; Peng et al., 2016c).

It was found that the chalky endosperm has starch grains loosely arranged and filled with a large number of bubbles in its endosperm. However, the starch granules were compact and fairly regular observed by SEM in the control rice endosperm ( $\mathrm{Li}$ et al., 2014). The results of our previous research showed, there were mainly four types of cell arrangement on the endosperm cross sections of different japonica rice varieties in southern Henan, corresponding to the varieties with white core or white belly in rice grain, the varieties without chalkiness and the varieties with white core and white belly observed by SEM in japonica grain (Peng et al., 2016a, 2016b). Further research by SEM showed that starch granules in chalkiness endosperm were not evenly distributed in japonica rice varieties from South Henan province, and the distribution of starch granules in the central part of the endosperm and in the dorsal-ventral direction is relatively concentrated (Figure 3). However, the starch granules of the endosperm in japonica rice varieties from South Henan province with no chalky character were evenly distributed throughout the endosperm, and the appearance of starch granules was relatively regular and starch granules were arranged closely. Therefore, the density of starch granules was larger. In different fragrant rice varieties (Peng et al., 2016b), it was also observed by SEM that the starch granules of fragrant rice grain were mostly ellipsoidal or spherical in the site of chalky endosperm; the starch granules were relatively small in diameter and inconsistent. It could also be observed that the gap between the starch granules inside the chalky endosperm of the fragrant rice varieties was relatively large, and the starch granules were mostly present as a single starch grain, so the arrangement of the starch granules was loose and filled with more bubble. Based on our previous research results ( $\mathrm{Li}$ et al., 2014b; Peng et al., 2016a, 2016b), there is a clear correlation between the pattern of cell arrangement and starch granules, the development of endosperm cells and chalky endosperm observed by SEM in rice, which will provide important information for revealing the mechanism of rice chalky traits and the improvement of rice grain quality. 

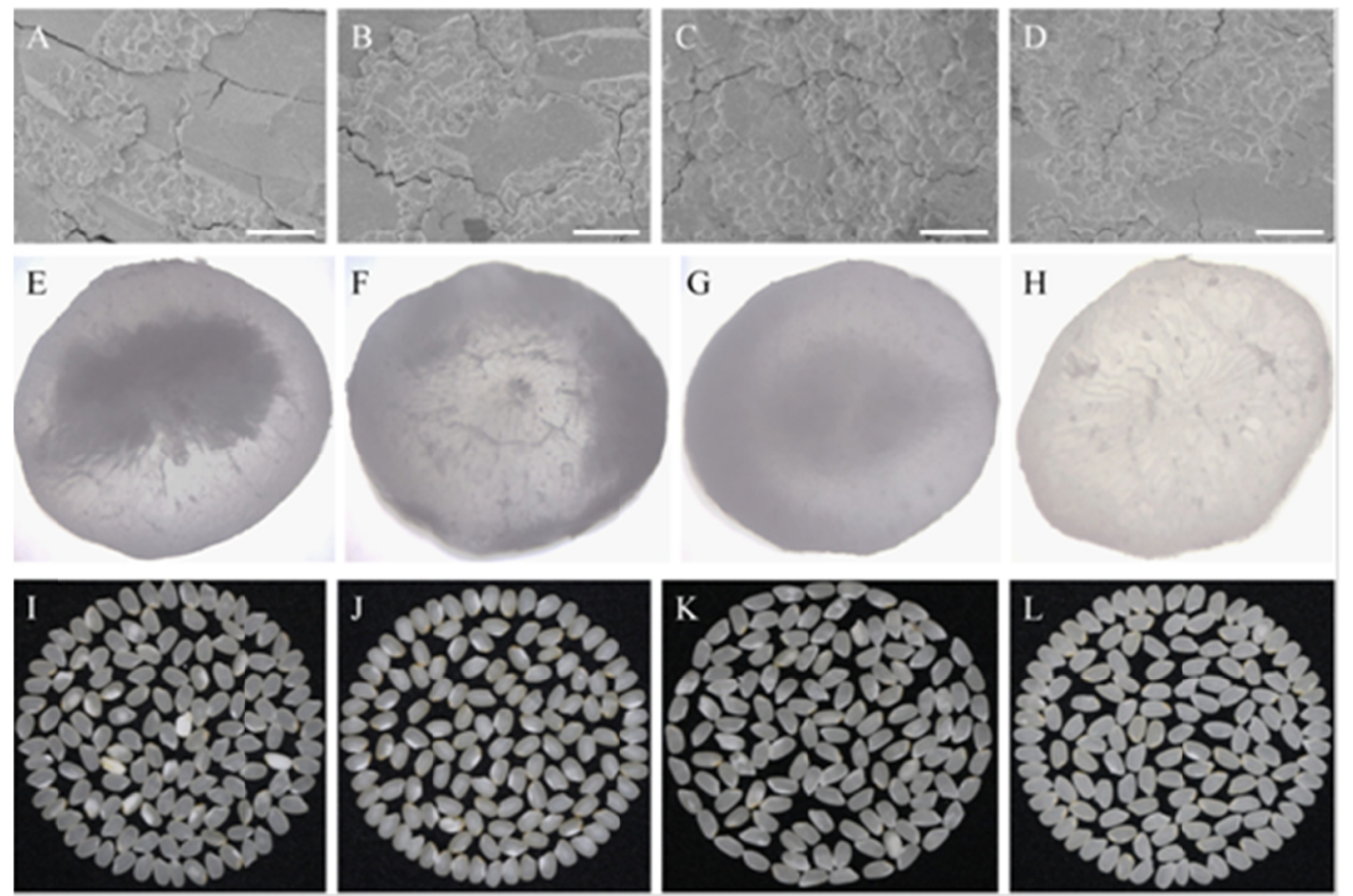

Figure 3. Low magnification view of the transversely fractured midregion of the tested rice

Note. A, E, I: Xianggeng 33; B, F, J: Longgeng 21; C, G, K: Longyou 1875; D, H, L: Xindao 18; Bar = $50 \mu \mathrm{m}$.

\section{Application of Near-Infrared Spectroscopy Technology in Genetic Improvement of Rice Grain Quality}

Modern near-infrared spectroscopy (NIRS) technology is a pollution-free, fast, non-destructive and high-throughput detection technology, and NIRS is a high-tech analysis technology that has been rapidly developed in the field of quality inspection and analysis of rice grain and the other major food crops in recent years (Bagchi et al., 2016). In the 1950s, the NIRS technology was first applied to the quality inspection of agricultural products, so that the application of NIRS analysis technology was gradually carried out in agricultural related fields (Molano et al., 2016). The NIRS analysis technology mainly includes three parts: near-infrared spectrometer, stoichiometric software and application model. Among them, the quantitative correction in NIRS detection analysis mainly includes principal component regression (PCR), partial least square method (PLS), multiple linear regression (MLR), stepwise regression (SMR), principal component analysis (PCA) and other methods (Bagchi et al., 2016). Using NIRS analysis technology, only the near-infrared spectra of rice samples could be scanned (Cossel et al., 2010; Luo et al., 2016), the quantitative or qualitative detection and analysis of rice grain quality and related characters could be carried out quickly according to the NIRS model.

The use of traditional technologies to detect physical and chemical indicators of rice grain quality has the disadvantages of low efficiency, complicated sample preparation, high cost, rigorous test process and irreversible damage to seeds (Cossel et al., 2010). However, there are many obvious advantages for the detection and analysis of the grain quality and its related traits using NIRS analysis technology in rice and other food crops: (1) A number of quality traits can be measured and analyzed at the same time; (2) Rice samples need not be pretreated to avoid damage caused by physical or chemical processes, i.e. non-destructive testing; (3) Automatic detection of rice grain samples, save a lot of manpower, material resources and no pollution; (4) The time required for NIRS instrument to sample is short and the detection speed is fast; (5) The skills of the near-infrared instruments for the operator are not high, and they are easy to train and popularize; (6) After the establishment of the mathematical model, it has good prediction effect and good reproducibility (Sohn et al., 2009; Ye et al., 2014; Cossel et al., 2010; Bagchi et al., 2016). Based on these advantages, NIRS analysis technology has made important progress in the detection of grain quality in rice, and its detection and analysis technologies have also been widely used in rice and other main grain crops. 
At present, NIRS technology has been relatively mature for the detection and analysis of the composition and content of the main components in rice grain, and the detection and analysis of micro-substance content in rice has also been successfully reported. The use of NIRS technology allows rapid and simultaneous detection of multiple quality traits and their associated traits in rice, including moisture, protein content, amylose content, total starch content, ash content, cadmium content and taste value, etc. (Bagchi et al., 2016; Wang et al., 2014; Peng et al., 2014; Zhu et al., 2015; Liu et al., 2016). Spectral data of different rice varieties with large differences in resistant starch content were collected and the mathematical model for predicting resistant starch content was constructed using the partial least squares method with the collected spectral data and the determined chemical value data (Luo et al., 2016), and then verified the mathematical model (including internal verification and external verification) to detect the content of resistant starch in rice, and the effect was better than traditional detection method. Near-infrared reflectance spectroscopy could be also used to predict the total amino acid content in brown rice and the contents of 13 amino acids in rice grain (Zhang et al., 2011). The use of NIRS analysis technology could predict the amino acid composition and total nitrogen content of rice flour, and there was a high correlation coefficient between amino acid content prediction results and traditional detection values (Wu et al., 2002; Lu et al., 2007). Using NIRS technology to predict total contents of phenol, flavonoid and antioxidant capacity in brown rice, established a calibration model to better predict the total phenol content and the antioxidant capacity of brown rice (Zhang et al., 2008; Kim et al., 2013). Rice oil was mixed with soybean oil, corn oil, rapeseed oil, restaurant waste oil, etc., respectively, and adulterated oil samples were prepared according to different proportions, then spectral collection of the mixed oil samples was performed by laser near-infrared spectroscopy ( $\mathrm{Tu}$ et al., 2015), qualitative models and quantitative models were established simultaneously, and the accuracy of predicted samples was relatively high. Thus, modern NIRS technology can not only quickly and qualitatively detect rice oil adulteration (Tyburczy et al., 2012), but also can be used as an important technology for the detection and analysis of other oils adulterants.

The detection and analysis of rice quality in storage, processing and distribution have attracted much attention. NIRS analysis technology can quickly and accurately detect and analyze the indicators such as germination rate of rice seeds, storage time of aged rice and fresh rice. Grains of different hybrid rice varieties were aged for different lengths of time through artificial accelerated aging methods, and their spectral data were collected by near-infrared spectroscopy, then a corresponding mathematical model was established and verified, and the results showed that the error between the predicted value and the actual value was very small by NIRS analysis technology (Cossel et al., 2010), indicating that the NIRS analysis technology could rapidly and nondestructively detect the germination rate of rice seeds. Using NIRS analysis combined with nuclear partial least squares to establish a coupled mathematical model between rice spectral characteristics and storage time, which could be used for rapid detection and analysis of different rice storage periods (Ye et al., 2014; Guo et al., 2013). The two different rice samples were blended according to different proportions, then the spectral images were collected by NIRS analysis technology, and finally, a mathematical model was established for quantitative analysis of rice with different grades of rice grain quality (Luo et al., 2016; Zhou et al., 2016; Huang et al., 2012a; Feng et al., 2013). This will provide a rapid and accurate scientific detection method for quantitative detection and discrimination of adulterated rice grains. Therefore, high and new technology has been widely used in the field of rice quality detection and analysis, and has become a modern detection and analysis technology which is developing faster and more and more noticeable.

\section{Application of Other High-Tech Technologies in Genetic Improvement of Rice Grain Quality}

Traditional methods for the determination of physical and chemical indicators of rice grain quality have many deficiencies such as the complicated grain sample pretreatment, low efficiency, high cost, rigorous test procedures, and they often cause irreversible damage to rice seeds (Cossel et al., 2010). With the rapid development of modern science and technology, various high and new technologies are continuously applied to the detection and improvement of rice grain quality to make up for the inadequacies of traditional detection and analysis methods. In addition to the above mentioned high-tech technologies (such as high-throughput sequencing, genome editing technology, NIRS technology and SEM technology), there are also some high-tech technologies have been used for genetic improvement of rice grain quality, such as electronic nose detection technology, computer vision technology, texture analysis technology, differential scanning calorimetry technology and so on (Jin et al., 2015; Xu et al., 2014; Park et al., 2012; Parnsakhorn et al., 2012; Zheng et al., 2009; Roberto et al., 2006; Zhou et al., 2010; Yu et al., 2009; Thorpe et al., 2010). Electronic nose detection technology could rapidly detect and analyze odor molecules in rice grains based on the contact of odor molecules with metal oxides or biofilms (Zheng et al., 2009). At the same time, electronic nose detection technology colud also be used to predict fungal infections in grains and even the extent of contamination (Jin et al., 2015; Roberto 
et al., 2006). Differential scanning calorimetry technology has also been reported to detect the gelatinization temperature of starch in rice. For example, the degree of starch retrogradation in rice could be expressed by the change of the enthalpy value, if the amylose content in rice was higher, the corresponding change of enthalpy value would be greater too (Zhou et al., 2010; Yu et al., 2009). It is noteworthy that computer vision technology has been widely used in the detection and analysis of rice appearance quality traits. For example, computer vision technology could be used to quickly determine the chalky grain rate and chalkiness area in rice, and it could also be used to distinguish pure rice, whole rice, and even the different rice varieties (Ola et al., 2013; Zhou et al., 2010; Ren et al., 2014), which had greatly facilitated the improvement of rice appearance quality. Therefore, with the rapid development of detection technologies, various high-tech technologies are continuously applied to the detection and analysis of rice grain quality, which will certainly bring great convenience to the genetic improvement of rice grain quality.

\section{Prospect of the Application of High and New Technology in Genetic Improvement of Rice Grain Quality}

Rice grain quality is a complex quantitative trait, consisting mainly of processing quality, appearance quality, nutritional quality, cooking and eating quality, etc. (Tsukaguchi et al., 2016; Peng et al., 2016a; Bhullar et al., 2013; Peng et al., 2014b). The detection, analysis, and evaluation of grain quality is crucial in rice genetic breeding program. Various high and new technologies have been used in the detection and analysis of rice grain quality, such as high-throughput sequencing technology, genome editing technology, scanning electron microscopy technology, NIRS technology, electronic nose detection technology, computer vision technology, and texture analysis technology, differential scanning calorimetry technology and the other high-tech technologies. These mordern technologies are the further complement and perfection of the traditional technologies for the detection and analysis in rice grain quality, but they all have their own advantages and disadvantages in the practical application of rice quality improvement. With the continuous emergence of new instruments and equipments and the gradual improvement of rice grain quality detection and analysis methods, a variety of non-destructive technologies have gradually been used in the detection and analysis of rice grain quality, and they are rapidly developed and have a wide range of applications. At present, the high and new technologies have covered the quality detection and analysis of rice grain in production, storage, processing, circulation and other links, such as the modern near infrared spectroscopy technology and computer vision technology, etc. Especially in the rice circulation process, it should be able to achieve rapid quality detection and quality control of rice and trace it back. Therefore, for the detection and improvement of rice grain quality, the future development trend is necessary to develop the universal and simple-type equipment in terms of testing equipment. Simultaneously, in the detection and analysis field, it able to achieve a unified set of detection and analysis methods, which should have the characteristics of good accuracy, short time, fast detection speed, stable performance and simple operation.

\section{Conclusion}

Rice is considered as one of the most important nourishment crops worldwide, and it has great scientific significance and practical application value for the improvement of the quality of rice grain using high and new technologies. At present, the use of high and new technologies in the genetic improvement of grain quality have made a series of important progress; for instance, the techniques of high throughput sequencing, genomic editing, near infrared spectroscopy, scanning electron microscopy, and other new ones have been widely apllied in the aim of the genetic improvement of grain quality in rice. Moreover, we can expect that, the high and new technologies are the further complement and perfection of the traditional technologies for the detection and analysis in rice grain quality. Indeed, the new technologies have covered the quality detection and analysis of rice grain in production, storage, processing, circulation and other links. Though, the future development trend is necessary to develop the universal and simple-type equipment in terms of testing equipment, and it able to achieve a unified set of detection and analysis methods applied to genetic improvement of grain quality in rice.

\section{Acknowledgements}

This work was financially supported by National Natural Science Foundation of China (U1604110, U1404319, 31600992, 31801332), Key Project of Science and Technology in Henan Province (182102110442), Major Science and Technology Project in Henan Province (121100110200), Key Project of Innovation and Entrepreneurship for Undergraduate in Henan Province (S201810477004), Nanhu Scholars Program for Young Scholars of XYNU (2016054), Scientific Research Innovation Project for Postgraduate of XYNU (2017KYJJ44), Student Research Fund Project of XYNU (2018-DXS-066), Key Scientific Research Projects of Universities in Henan Province (19A180030) and Institute for Conservation and Utilization of Agro-bioresources in Dabie Mountains. 


\section{References}

Ansorge, W. J. (2009). Next-generation DNA sequencing techniques. New Biotechnology, 25(4), $195-203$. https://doi.org/10.1016/j.nbt.2008.12.009

Araikichise, Y., Shiwa, Y., Nagasaki, H., Ebana, K., Yoshikawa, H., Yano, M., \& Wakasa, K. (2011). Discovery of genome-wide DNA polymorphisms in a landrace cultivar of japonica rice by whole-genome sequencing. Plant and Cell Physiology, 52(2), 274-282. https://doi.org/10.1093/pcp/pcr003

Bagchi, T. B., Sharma, S., \& Chattopadhyay, K. (2016). Development of NIRS models to predict protein and amylose content of brown rice and proximate compositions of rice bran. Food Chemistry, 191, 21-27. https://doi.org/10.1016/j.foodchem.2015.05.038

Bhullar, N. K., \& Gruissem, W. (2013). Nutritional enhancement of rice for human health: The contribution of biotechnology. Biotechnology Advances, 31, 50-57. https://doi.org/10.1016/j.biotechadv.2012.02.001

Birla, D. S., Malik, K., Sainger, M., Chaudhary, D., Jaiwal, R., \& Jaiwal, P. K. (2017). Progress and challenges in improving the nutritional quality of rice (Oryza sativa L.). Critical Reviews in Food Science and Nutrition, 57(11), 2455-2481. https://doi.org/10.1080/10408398.2015.1084992

Chen, Y., Wang, M., \& Ouwerkerk, P. B. F. (2013). Molecular and environmental factors determining grain quality in rice. Food and Energy Security, 1, 111-132. https://doi.org/10.1002/fes3.11

Cossel, K. C., Adler, F., Maslowski, P., \& Ye, J. (2010). Applications of cavity- enhanced direct frequency comb spectroscopy. Annual Review of Analytical Chemistry, 8011(1), 581-584.

Feng, Z., Zhang, B., Ding, W., Liu, X., Yang, D. L., Wei, P., ... Zhu, J. K. (2013). Efficient genome editing in plants using a CRISPR/Cas system. Cell Research, 23(10), 1229-1232. https://doi. org/10.1038/cr.2013.114

Gaj, T., Gersbach, C. A., \& Barbas, C. F. (2013). ZFN, TALEN, and CRISPR/ Cas-based methods for genome engineering. Trends in Biotechnology, 31(7), 397-405. https://doi.org/10.1016/j.tibtech.2013.04.004

Gao, F., Liu, J., Yang, L., Wu, X., Xiao, Y., Xia, X., \& He, Z. (2016). Genome-wide linkage mapping of QTL for physiological traits in a Chinese wheat population using the 90K SNP array. Euphytica, 209(3), 789-804. https://doi.org/10.1007/s10681-016-1682-6

Gao, Y., Xue, D. W., Qian, Q., \& Gao, Z. Y. (2015). Application of the second generation sequencing technology in rice genomics and transcriptomics. Chinese Journal of Rice Science, 29(2), 208-214. https://doi.org/10.3969/j.issn.1001-7216.2015.02.013

Gil-Humanes, J., Wang, Y., Liang, Z., Shan, Q., Ozuna, C. V., Sánchez-León, S., ... Voytas, D. F. (2017). High-efficiency gene targeting in hexaploid wheat using DNA replicons and CRISPR/Cas9. Plant Journal, 89(6), 1251-1262. https://doi.org/10.1111/tpj.13446

Guo, Y., Cai, W., Tu, K., Tu, S., Wang, S., Zhu, X., \& Zhang, W. (2013). Infrared and raman spectroscopic characterization of structural changes in albumin, globulin, glutelin, and prolamin during rice aging. Journal of Agricultural and Food Chemistry, 61(1), 185-92. https://doi.org/10.1021/jf303345r

He, Q., \& Park, Y. J. (2015). Discovery of a novel fragrant allele and development of functional markers for fragrance in rice. Molecular Breeding, 35(11), 1-10. https://doi.org/10.1007/s11032-015-0412-4

Huang, L., Li, Z., Wu, J., Xu, Y., Yang, X., Fan, L., ... Zhou, X. (2015). Analysis of genetic variation and diversity of Rice stripe virus populations through high-throughput sequencing. Frontiers in Plant Science, 6 , 176. https://doi.org/10.3389/fpls.2015.00176

Huang, X., Kurata, N., Wei, X., Wang, Z. X, Wang, A., Zhao, Q., .. Han, B. (2012a). A map of rice genome variation reveals the origin of cultivated rice. Nature, 490(7421), 497-501. https://doi.org/10.1038/ nature 11532

Huang, X., Wei, X., Sang, T., Zhao, Q., Feng, Q., Zhao, Y., ... Han, B. (2010). Genome-wide association studies of 14 agronomic traits in rice landraces. Nature Genetics, 42(11), 961-967. https://doi.org/10.1038/ng.695

Huang, X., Zhao, Y., Wei, X., Li, C., Wang, A., Zhao, Q., ... Han, B. (2012b). Genome-wide association study of flowering time and grain yield traits in a worldwide collection of rice germplasm. Nature Genetics, 44(1), 32-39. https://doi.org/10.1038/ng.1018

Jin, J., Deng, S., Ying, X., Ye, X., Lu, T., \& Hui, G. (2015). Study of herbal tea beverage discrimination method using electronic nose. Journal of Food Measurement and Characterization, 9(1), 52-60. https://doi.org/ 10.1007/s11694-014-9209-0 
Johnson, R. A., Gurevich, V., Filler, S., Samach, A., \& Levy, A. A. (2015). Comparative assessments of CRISPR-Cas nucleases' cleavage efficiency in planta. Plant Molecular Biology, 87(1), 143-156. https://doi.org/10.1007/s11103-014-0266-x

Kang, H. Q., \& Chang, H. Y., (2007). Chalky traits and endosperm structure of hybrid rice major parental materials. Chinese Agricultural Science Bulletin, 23(4), 180-185.

Kim, J. K., Park, S. Y., Lim, S. H., Yeo, Y., Cho, H. S., \& Ha, S. H. (2013). Comparative metabolic profiling of pigmented rice (Oryza sativa L.) cultivars reveals primary metabolites are correlated with secondary metabolites. Journal of Cereal Science, 57(1), 14-20. https://doi.org/10. 1016/j.jcs.2012.09.012

Kovach, M. J., Calingacion, M. N., Fitzgerald, M. A., \& Mccouch, S. R. (2009). The origin and evolution of fragrance in rice (Oryza sativa L.). Proceedings of the National Academy of Sciences USA, 106(34), 14444-14449. https://doi.org/10.1073/pnas.0904077106

Kusano, M., Yang, Z., Okazaki, Y., Nakabayashi, R., Fukushima, A., \& Saito, K. (2015). Using metabolomic approaches to explore chemical diversity in rice. Molecular Plant, 8, 58-67. https://doi.org/10.1016/j.molp. 2014.11.010

Li, J. Y., Wang, J., \& Zeigler, R. S. (2014a). The 3,000 rice genomes project: New opportunities and challenges for future rice research. Giga Science, 3(1), 1-3. https://doi.org/10.1186/2047-217X-3-8

Li, M., Li, X., Zhou, Z., Wu, P., Fang, M., Pan, X., ... Li, H. (2016). Reassessment of the four yield-related genes Gn1a, DEP1, GS3, and IPA1 in rice using a CRISPR/Cas9 system. Frontiers in Plant Science, 7, 377. https://doi.org/10.3389/fpls.2016.00377

Li, T., Liu, B., Spalding, M. H., Weeks, D. P., \& Yang, B. (2012). High-efficiency TALEN-based gene editing produces disease-resistant rice. Nature Biotechnology, 30, 390-392. https://doi.org/10.1038/nbt.2199

Li, X., Zhou, W., Ren, Y., Tian, X., Lv, T., Wang, Z., ... Bu, Q. (2017). High-efficiency breeding of early-maturing rice cultivars via CRISPR/Cas9-mediated genome editing. Journal of Genetics and Genomics, 44(3), 175-178. https://doi.org/10.1016/j.jgg.2017.02.001

Li, Y., Fan, C., Xing, Y., Yun, P., Luo, L., Yan, B., ... He, Y. (2014b). Chalk5 encodes a vacuolar $\mathrm{H}+$-translocating pyrophosphatase influencing grain chalkiness in rice. Nature Genetics, 46(4), 398-404. https://doi.org/10.1038/ng.2923

Liang, Z., Zhang, K., Chen, K., \& Gao, C. (2014). Targeted mutagenesis in Zea mays using TALENs and the CRISPR/Cas system. Journal of Genetics and Genomics, 41, 63-68. https://doi.org/10.1016/j.jgg.2013. 12.001

Liu, H., Ding, Y., Zhou, Y., Jin, W., Xie, K., \& Chen, L. L. (2017). CRISPR-P 2.0: An improved CRISPR-Cas9 tool for genome editing in plants. Molecular Plant, 10(3), 530-532. https://doi.org/10.1016/j.molp. 2017.01.003

Liu, K., Sun, M. F., Yan, G. H., \& Tang, H. S. (2016). Near infrared spectroscopy assisted selection of rice taste quality breeding. Journal of Jiangsu Agricultural Sciences, 44(7), 121-122. https://doi.org/10.15889/j.issn. 1002-1302.2016.07.032

Liu, Y., Yu, X. Q., Zhang, A. N., Wang, F. M., \& Liu, G. L. (2016). Application of high throughput genome sequencing in crop gene location and excavation. Journal of Shanghai Agricultural Sciences, 32(6), 171-175. https://doi.org/10.15955/j.issn.1000-3924.2016.06.32

Lu, Q., Chen, Y., Mikami, T., Kawano, M., \& Li, Z. (2007). Adaptability of four-samples sensory tests and prediction of visual and near-infrared reflectance spectroscopy for Chinese indica rice. Journal of Food Engineering, 79(4), 1445-1451. https://doi.org/10.1016/j.jfoodeng.2006.04.046

Luo, X., Wu, F. X., Xie, H. G., Zhu, Y. S., Zhang, J. F., \& Xie, H. A. (2016). Study on determination of resistant starch content in rice by near infrared spectroscopy. Spectroscopy and Spectral Analysis, 36(3), 697-701. https://doi.org/10.3964/j.issn.1000-0593(2016)03-0697-05

Ma, X., Zhang, Q., Zhu, Q., Liu, W., Chen, Y., Qiu, R., ... Liu, Y. G. (2015). A robust CRISPR/Cas9 system for convenient, high-efficiency multiplex genome editing in monocot and dicot plants. Molecular Plant, 8, 1274-1284. https://doi.org/10.1016/j.molp.2015.04.007

Mao, Y., Zhang, H., Xu, N., Zhang, B., Gou, F., \& Zhu, J. K. (2013). Application of the CRISPR-Cas system for efficient genome engineering in plants. Molecular Plant, 6, 2008-2011. https://doi. org/10.1093/mp/sst121 
Miao, J., Guo, D., Zhang, J., Huang, Q., Qin, G., Zhang, X., ... Qu, L. J. ( 2013). Targeted mutagenesis in rice using CRISPR-Cas system. Cell Research, 23(10), 1233-1236. https://doi.org/10.1038/cr.2013.123

Molano, M. L., Cortes, M. L., Avila, P., Martens, S. D., \& Munoz, L. S. (2016). Near infrared spectroscopy (NIRS) calibration equations to predict nutritional quality parameters of tropical forages. Tropical Grasslands-Forrajes Tropicales, 4(3), 139-145. https://doi.org/10.17138/TGFT(4)139-145

Noman, A., Aqeel, M., \& He, S. (2016). CRISPR-Cas9: Tool for qualitative and quantitative plant genome editing. Frontiers in Plant Science, 7(30910), 1740. https://doi.org/10.3389/fpls.2016.01740

Ola, D., Manescu, M., Cristea, L., Budde, J., \& Hoffmann, T. (2013). Software application in machine vision investigation of agricultural seeds quality. Applied Mechanics and Materials, 436, 463-473. https://doi.org/ 10.4028/www.scientific.net/AMM.436.463

Pandey, M. K., Rani, N. S., Madhav, M. S., Sundaram, R. M., Varaprasad, G. S., Sivaranjani, A. K., ... Kumar, A. (2012). Different isoforms of starch-synthesizing enzymes controlling amylose and amylopectin content in rice (Oryza sativa L.). Biotechnology Advances, 30, 1697-1706. https://doi.org/10.1016/j.biotechadv. 2012.08.011

Park, C. E., Kim, Y. S., Park, K. J., \& Kim, B. K. (2012). Changes in physicochemical characteristics of rice during storage at different temperatures. Journal of Stored Products Research, 48, 25-29. https://doi.org/ 10.1016/j.jspr.2011.08.005

Parnsakhorn, S., \& Noomhorm, A. (2012). Effects of storage temperature on physical and chemical properties of brown rice, parboiled brown rice and parboiled paddy. Thai Journal of Agricultural Science, 45(4), 221-231.

Peng, B., Kong, H. L., Li, Y. B., Wang, L. Q., Zhong, M., Sun, L., ... He, Y. Q. (2014a). OsAAP6 functions as an important regulator of grain protein content and nutritional quality in rice. Nature Communications, 5 , 4847. https://doi.org/10.1038/ncomms5847

Peng, B., Pang, R. H., Sun, Y. F., Geng, L. P., Song, X. Q., Li, H. L., ... Song, S. Z. (2016). Chalkiness characters and endosperm scanning electron microscope observation of rice grain in different fragrant varieties. Journal of Southern Agriculture, 29(10), 1635-1641. https://doi.org/10.3969/j.issn.2095-1191. 2016.10.163

Peng, B., Sun, Y. F., Li, Q. R., Li, D., Pang, R. H., Zhou, Q. Y., ... Song, S. Z. (2016). Progress in genetic research on rice chalkiness. Journal of Xinyang Normal University (Natural Science Edition), 29(2), 304-312. https://doi.org/10.3969/j.issn.1003-0972.2016.02.035

Peng, B., Sun, Y. F., Pang, R. H., Li, H. L., Song, X. H., Yuan, H. Y., ... Song, S. Z. (2016). Studies on chalky characters of different japonica rice cultivars and their endosperm structure observation. Journal of Zhejiang Agricultural Sciences, 28(11), 1803-1811. https://doi.org/10.3969/j.issn.1004-1524.2016.11.01

Peng, B., Wang, L., Fan, C., Jiang, G., Luo, L., Li, Y., \& He, Y. (2014b). Comparative mapping of chalkiness components in rice grain using five populations across two environments. BMC Genetics, 15, 49. https://doi.org/10.1186/1471-2156-15-49

Ren, G. Y., Li, X. J., Yin, J., Zhang, C. F., Wang, G. L., \& Zhang, Z. J., (2014). Research progress and prospect of rice quality testing technology. Chinese Journal of Grain and Oil, 29(2), 115-120.

Roberto, P., Adriano, A., Eugenio, M., Corrado, D., Arnaldo, D., Maria, G., ... Corrado, F. (2006). Detection of fungal contamination of cereal grain sample by an electronicnose. Sensors and Actuators B: Chemical, 119(2), 425-430. https://doi.org/10.1016/j.snb.2005.12.047

Samanta, M. K., Dey, A., \& Gayen, S. (2016). CRISPR/Cas9: An advanced tool for editing plant genomes. Transgenic Research, 25(5), 561-573. https://doi.org/10.1007/s11248-016-9953-5

Shan, Q., Zhang, Y., Chen, K., Zhang, K., \& Gao, C. (2015). Creation of fragrant rice by targeted knockout of the OsBADH2 gene using TALEN technology. Plant Biotechnology Journal, 13, 791-800. https://doi.org/ 10.1111/pbi.12312

Shapter, F. M., Cross, M., Ablett, G., Malory, S., Chivers, I. H., King, G. J., \& Henry, R. J. (2013). High-throughput sequencing and mutagenesis to accelerate the domestication of Microlaena stipoides as a new food crop. PLoS One, 8(12), e82641. https://doi.org/10.1371/journal.pone.0082641 
Shen, L., Wang, C., Fu, Y., Wang, J., Liu, Q., Zhang, X., ... Wang, K. (2018). QTL editing confers opposing yield performance in different rice varieties. Journal of Integrative Plant Biology, 60(2), 89-93. https://doi.org/10.1111/jipb.12501

Sohn, M. Y., Kim, Y. K., Vines, L. L., \& Kays, S. E. (2009). Near infrared analysis of lipid classes in processed cereal products. Journal of Near Infrared Spectroscopy, 17(3), 127-133. https://doi. org/10.1255/jnirs.835

Su, N., Hu, M. L., Wu, D. X., Wu, F. Q., Fei, G. L., Lan, Y., ... Wan, J. M. (2012). Disruption of a rice pentatricopeptide repeat protein causes a seedling-specific albino phenotype and its utilization to enhance seed purity in hybrid rice production. Plant Physiology, 159(1), 227-38. https://doi.org/10.1104/pp.112. 195081

Subbaiyan, G. K., Waters, D. L. E., Katiyar, S. K., Sadananda, A. R., Vaddadi, S., \& Henry, R. J. (2012). Genome-wide DNA polymorphisms in elite indica rice inbreds discovered by whole-genome sequencing. Plant Biotechnology Journal, 10(6), 623-634. https://doi.org/10.1111/j.1467-7652.2011.00676.x

Tatarinova, T. V., Chekalin, E., Nikolsky, Y., Bruskin, S., Chebotarov, D., McNally, K. L., \& Alexandrov, N. (2016). Nucleotide diversity analysis highlights functionally important genomic regions. Scientific Reports, 6, 35730. https:// doi.org/10.1038/srep35730

Thorpe, M. J., \& Ye, J. (2008). Cavity-enhanced direct frequency comb spectroscopy. Applied Physics B, 91(3), 397-414. https://doi.org/10.1146/annurev-anchem-060908-155248

Tsukaguchi, T., Taniguchi, Y., \& Ito, R. (2016). The effects of nitrogen uptake before and after heading on grain protein content and the occurrence of basal- and back-white grains in rice (Oryza sativa L.). Plant Production Science, 19(4), 508-517. https://doi.org/10.1080/1343943X.2016.1223527

Tu, B., Song, Z. Q., Zheng, X., Zeng, L. L., Yin, C., He, D. P., \& Qi, P. S. (2015). 2015. Quantitative analysis of rice oil adulteration based on laser near infrared. Spectroscopy and Spectral Analysis, 35(6), 1539-1545. https://doi.org/10.3964/j.issn.1000-0593(2015)06-1539-07

Tyburczy, C., Mossoba, M. M., Fardin-Kia, A. R., \& Rader, J. I. (2012). Evaluation of low transfat edible oils by attenuated total reflection-fourier transform infrared spectroscopy and gas chromatography: A comparison of analytical approaches. Analytical and Bioanalytical Chemistry, 404(3), 809-819. https://doi.org/ 10.1007/s00216-012-6189-y

Wang, C., Shen, L., Fu, Y., Yan, C., \& Wang, K. (2015). A simple CRISPR/Cas9 system for multiplex genome editing in rice. Journal of Genetics and Genomics, 42, 703-706. https://doi.org/10.1016/j.jgg.2015.09.011

Wang, L., Liu, D., Pu, H., Sun, D. W., Gao, W., \& Xiong, Z. (2015). Use of hyperspectral imaging to discriminate the variety and quality of rice. Food Analytical Method, 8, 515-523. https://doi.org/ 10.1007/s12161-014-9916-5

Wang, L., Wang, A., Huang, X., Zhao, Q., Dong, G., Qian, Q., ... Han, B. (2011). Mapping 49 quantitative trait loci at high resolution through sequencing-based genotyping of rice recombinant inbred lines. Theoretical and Applied Genetics, 122(2), 327-340. https://doi.org/10.1007/s00122-010-1449-8

Wang, Y., Cheng, X., Shan, Q., Zhang, Y., Liu, J., Gao, C., \& Qiu, J. L. (2014). Simultaneous editing of three homoeo-alleles in hexaploid bread wheat confers heritable resistance to powdery mildew. Nature Biotechnology, 32, 947-951. https://doi.org/10.1038/nbt.2969

Wendt, T., Holm, P. B., Starker, C. G., Christian, M., Voytas, D. F., Brinchpedersen, H., \& Holme, I. B. (2013). TAL effector nucleases induce mutations at a pre-selected location in the genome of primary barley transformants. Plant Molecular Biology, 83, 279-285. https://doi.org/10.1007/s11103-013-0078-4

Wu, J. G., Shi, C., \& Zhang, X. (2002). Estimating the amino acid composition in milled rice by near-infrared reflectance spectroscopy. Field Crops Research, 75(1), 1-7. https://doi.org/10.1016/S0378-4290(02) 00006-0

Xu, R. F., Li, H., Qin, R. Y., Li, J., Qiu, C. H., Yang, Y. C., ...Yang, J. B. (2015). Generation of inheritable and "transgene clean" targeted genome-modified rice in later generations using the CRISPR/Cas9 system. Scientific Reports, 5, 11491. https://doi.org/10.1038/srep11491

$\mathrm{Xu}, \mathrm{R}$., Wei, P., \& Yang, J. (2017). Use of CRISPR/Cas genome editing technology for targeted mutagenesis in rice. Methods in Molecular Biology, 1498, 33. https://doi.org/10.1007/978-1-4939-6472-7-3 
Xu, R., Yang, Y., Qin, R., Hao, L., Qiu, C., Li, L., ... Ang, J. (2016). Rapid improvement of grain weight via highly efficient CRISPR/Cas9-mediated multiplex genome editing in rice. Journal of Genetics and Genomics, 43(8), 529. https://doi.org/10.1016/j.jgg.2016.07.003

Xu, S., Zhou, Z., Lu, H., Luo, X., Lan, Y., Zhang, Y., \& Li, Y. F. (2014). Estimation of the age and amount of brown rice plant hoppers based on bionic electronic nose use. Sensors, 14(10), 18114. https://doi.org/10.3390/s141018114

Xu, X., \& Bai, G. (2015). Whole-genome resequencing: Changing the paradigms of SNP detection, molecular mapping and gene discovery. Molecular Breeding, 35(1), 33. https://doi.org/10.1007/ s11032-015-0240-6

Ye, M., Yue, T., Yuan, Y., \& Li, Z. (2014). Application of FT-NIR spectroscopy to apple wine for rapid simultaneous determination of soluble solids content, $\mathrm{pH}$, total acidity, and total ester content. Food and Bioprocess Technology, 7(10), 3055-3062. https://doi.org/10.1007/s11947-014-1385-8

Yu, H., Xie, W., Wang, J., Xing, Y., Xu, C., Li, X., ... Zhang, Q. (2011). Gains in QTL detection using an ultra-high density SNP map based on population sequencing relative to traditional RFLP/SSR markers. PLoS One, 6(3), e17595. https://doi. org/10.1371/journal.pone.0017595

Yu, S. F., Ying, M., \& Sun, D. W. (2009). Impact of amylose content on starch retrogradation and texture of cooked milled rice during storage. Journal of Cereal Science, 50, 139-144. https://doi.org/10.1016/j.jcs. 2009.04.003

Zhang, B., Rong, Z. Q., Shi, Y., Wu, J. G., \& Shi, C. H. (2011). Prediction of the amino acid composition in brown rice using different sample status by near-infrared reflectance spectroscopy. Food Chemistry, 127(1), 275-281. https://doi.org/10.1016/j.foodchem.2010.12.110

Zhang, C. Q., Zhao, D. S., Li, Q. F., Gu, M. H., \& Liu, Q. Q. (2016). Advances in cloning and functional analysis of rice quality traits. Chinese Journal of Agricultural Sciences, 49(22), 4267-4283. https://doi.org/10.3864/j.issn.0578-1752.2016.22.002

Zhang, C., Shen, Y., Chen, J., Xiao, P., \& Bao, J. (2008). Nondestructive prediction of total phenolics, flavonoid contents, and antioxidant capacity of rice grain using near-infrared spectroscopy. Journal of Agricultural and Food Chemistry, 56(18), 8268-8272. https://doi.org/10.1021/jf801830z

Zhang, H., Zhang, J., Wei, P., Zhang, B., Gou, F., Feng, Z., ... Zhu, J. K. (2014). The CRISPR/Cas9 system produces specific and homozygous targeted gene editing in rice in one generation. Plant Biotechnology Journal, 12(6), 797-807. https://doi.org/10.1111/pbi.12200

Zheng, X. Z., Lan, Y. B., Zhu, J. M., Westbrook, J., Hoffmann, W. C., \& Lacey, R. E. (2009). Rapid identification of rice samples using an electronic nose. Journal of Bionic Engineering, 6(3), 290-297. https://doi.org/10.1016/S1672-6529(08)60122-5

Zhou, H., Bo, L., Weeks, D. P., Spalding, M. H., \& Bing, Y. (2014). Large chromosomal deletions and heritable small genetic changes induced by CRISPR/Cas9 in rice. Nucleic Acids Research, 42(17), 10903-10914. https:// doi.org/10.1093/nar/gku806

Zhou, W., Wang, X., Zhou, D., Ouyang, Y., \& Yao, J. (2016). Overexpression of the 16-kDa a-amylase/trypsin inhibitor RAG2 improves grain yield and quality of rice. Plant Biotechnology Journal, 15, 568-580. https://doi.org/10.1111/pbi.12654

Zhou, X. X., Xie, S. M., Chen, Q. S., \& Zhang, Z. Z. (2016). Quantitative discrimination of rice adulteration based on near infrared spectroscopy. Journal of Anhui Agricultural University, 43(4), 503-507. https://doi.org/10.13610/j.cnki.1672-352x.20160712.025

Zhou, Z. H., Peng, S. L., Bo, X. C., \& Li, F. (2017). Analysis of microbial detection data based on high-throughput sequencing technology. Advances in Biochemistry and Biophysics, 44(1), 58-69. https://doi.org/10.16476/j.pibb.2016. 0239

Zhou, Z., Robards, K., Helliwell, S., \& Blanchard, C. (2010). Effect of storage temperature on rice thermal properties. Food Research International, 43(3), 709-715. https://doi.org/10.1016/j.foodres.2009.11.002

Zhu, X. R., Li, G. Y., Su, D. L., Liu, W., Shan, Y., \& Branch, L. P. (2015). Rapid detection of cadmium content in rice based on near infrared spectroscopy and combined interval partial least squares method. Food and Machinery, 31(7), 43-46. https://doi.org/10.13652/j.issn.1003-5788.2015.04. 011 


\section{Copyrights}

Copyright for this article is retained by the author(s), with first publication rights granted to the journal.

This is an open-access article distributed under the terms and conditions of the Creative Commons Attribution license (http://creativecommons.org/licenses/by/4.0/). 